\title{
UN ARTISTA CORTESANO EN LA BARCELONA DE CARLOS III DE AUSTRIA: APUNTES PROSOPOGRÁFICOS SOBRE ANDREA VACCARO II*
}

\author{
RoBerto Quirós Rosado \\ Universidad Autónoma de Madrid \\ roberto.quiros@alumni.uam.es
}

\begin{abstract}
La diversidad de focos de introducción de la cultura de corte italiana en los inicios del siglo XVIII español obliga a indagar en la circulación de artistas napolitanos en la Barcelona de Carlos III de Austria. Bajo esta premisa, en el estudio se analiza la estancia catalana del desconocido pintor napolitano Andrea Vaccaro II, sus lazos con la Real Cámara y el ministerio español del monarca Habsburgo, así como la concesión de oficios en los tribunales de Nápoles como pago por servicios en palacio.

Palabras clave: Andrea Vaccaro II; Carlos III/VI de Austria; Isabel Cristina de Braunschweig-Wolfenbüttel; Barcelona; Nápoles; Guerra de Sucesión: Pintor de Cámara; Siglo XVIII.
\end{abstract}

\section{A COURT ARTIST IN BARCELONA DURING THE REIGN OF CHARLES III OF HABSBURG: PROSOPOGRAPHICAL NOTES ABOUT ANDREA VACCARO II}

At the beginning of the $18^{\text {th }}$ century Barcelona was a principal entry point for the introduction of Italian courtly culture in Spain during the brief reign of Carlos III of Habsburg. This study analyzes the presence of the little-known Neapolitan painter Andrea Vaccaro II in Cataluña, and his connections with the Royal Chamber and the Spanish Habsburg ministry, as well as the concession of offices in Reame as payment for palace services.

Key words: Andrea Vaccaro II; Charles III/VI of Habsburg; Elizabeth Christine of Braunschweig-Wolfenbüttel; Barcelona; Naples; War of Spanish Succession; Court painter; 18th century.

Oscurecida durante siglos, Barcelona, la corte de Carlos III, el último Habsburgo español, está siendo redescubierta como uno de los principales focos de la irrupción de la cultura italiana en la España setecentista. La causa directa se halla en la llegada, a lo largo de los años 1705 a 1713, de diplomáticos, aristócratas, militares, ministros y artistas de las más diversas naciones europeas, con particular abundancia de italianos que recalaron en busca de mercedes y privilegios con que ver premiada su lealtad a la Augustísima Casa. La influencia de este proceso en el plano cultural ya fue advertida por el erudito Carreras i Bulbena en 1902. Sin embargo, sólo desde el campo musicológico y, en los últimos años, a causa de los ecos histórico-políticos de 1714, se ha devuelto el interés por las artes y la cultura política de la Barcelona austriaca.

* El presente estudio se inserta dentro del proyecto de la Dirección General de Investigación del Ministerio de Economía y Competitividad Sociedad cortesana y redes diplomáticas: la proyección europea de la monarquía de España (1659-1725) [HAR2015-67069-P (MINECO/FEDER)]. 
Los estudios de Andrea Sommer-Matthis, Danièle Lipp y Laura Bernardini han llamado la atención sobre la relevancia de la Capilla y la Cámara del melómano Carlos III, si bien, a los artistas musicales se sumaron otros de igual relevancia en otros espacios de la producción y consumo cultural de corte ${ }^{1}$. De esta forma, el gusto clasicista (dentro de su barroquismo) de las tramoyas teatrales, arquitecturas y otras composiciones artísticas pudo desarrollarse con los diseños de Ferdinando Galli Bibbiena, las piezas de orfebrería y escultura de Konrad Rudolf y del escultor de Cámara Joseph Callejo y Aragón, y mediante la presencia de Joseph de Churriguera, el afamado arquitecto madrileño que en otoño de 1710 había jurado fidelidad al soberano Habsburgo durante su estancia en la corte castellana. El reconocimiento de éste al monarca fue premiado con el nombramiento como "trazador mayor de mis Obras Reales del Alcázar de Madrid para quando llegue el caso de estar a mi obediencia aquellos dominios", según reza la nómina regia de $1712^{2}$. Por contra, menos conocida es la pléyade de retratistas que plasmaron con sus pinceles la majestad de los soberanos durante los atribulados años del conflicto bélico ${ }^{3}$. Entre ellos descollaron dos napolitanos que consiguieron acceder sucesivamente a la mayor dignidad de entre sus colegas, la de pintor de la Real Cámara: Alessandro Castrioto y Andrea Vaccaro II.

Fue el mencionado Carreras i Bulbena quien primero advirtiese de la estancia barcelonesa de Andrea Vaccaro (Nápoles, 1668 - ¿Barcelona / Nápoles?, c. 1713). Hijo legítimo del pintor y empresario teatral Nicola y su esposa Anna Maria, y nieto del afamado pintor homónimo, gracias a las Vite de Bernardo De Dominici se conocen datos sobre su parentela y círculo pictórico ${ }^{4}$. Según el tratadista napolitano, su progenitor se había formado en el taller paterno mediante la copia de sus propias obras "ed alcune di picciole figure facendone da se, acquistò il nome di degno figliuolo di sì gran padre". Tras su paso por Roma y con la influencia de otros conocidos pintores, se dio a conocer en Parténope con pinturas de sátiros y ninfas alla maniera di Poussin y otros lienzos de temática religiosa ${ }^{5}$ :

\footnotetext{
${ }^{1}$ Carreras i Bulbena, 1902. Respecto a la música, ópera y las artes vinculadas al escenario teatral en la Barcelona carolina consúltese Sommer-Mathis, 2007, Lipp, 2007 y Bernardini, 2009b. Una sintética pero completa visión de los principales sujetos de la corte de Carlos III en Barcelona se encuentra en Kalmár, 1998. Una reflexión más amplia sobre el sistema cortesano barcelonés, con especial hincapié en la gobernanza de Italia, en Quirós Rosado, 2015a. Para los vínculos financieros entre la urbe catalana y el espacio cultural italiano, vid. Badosa i Coll, 2003. Son escasas las aproximaciones al modo de vida cortesano y ceremonial, como se detalla en Quirós Rosado, 2015b. Por último, cabe destacarse la monografía sobre la vida cotidiana de la urbe catalana durante el conflicto sucesorio a cargo de García Espuche, 2014. Para una panorámica general del conflicto sucesorio, véase el análisis de Albareda Salvadó, 2010, y la monografía centrada en la monarquía austriaca española de León Sanz, 2014. Sobre la administración carolina todavía es útil, aunque con matices, la obra de Voltes Bou, 1963.

${ }^{2}$ Para el caso del escenógrafo y arquitecto Galli Bibbiena, vid. Bernardini, 2009a. Sobre Rudolf, quien había iniciado la construcción de la puerta principal de la catedral de Valencia antes de su seguimiento del séquito del rey Carlos a Barcelona en 1707, Boix, 1845, p. 414. La noticia sobre el escultor Callejo, de quien se dice en el privilegio haber recalado en Barcelona siguiendo al monarca austriaco, se halla en AHN, Estado, legajo 8687. Título de Carlos III a favor de Joseph Callejo y Aragón, Barcelona, 25 de agosto de 1711.

${ }^{3}$ Para aproximarse al conocimiento de los gustos pictóricos barceloneses del Setecientos, con algunas pequeñas referencias a los artistas cortesanos de Carlos III, vid. Alcolea, 1959-1960. Para una mayor profundización sobre los pintores de Cámara y los gustos retratísticos de la corte madrileña del Seiscientos, vid. Pascual Chenel, 2010. Respecto a los pintores palatinos de Felipe $\mathrm{V}$ coetáneos a los barceloneses, entre los que se contaba un Nicola Vaccaro (homónimo al padre del pintor de Barcelona) activo en Madrid en la década de 1710 y sobre el que se desconoce la posible filiación con el Vaccaro carolino, véase Úbeda de los Cobos, 2002, pp. 110, 113.

${ }^{4}$ Carreras i Bulbena, 1902, pp. 221-223.

${ }^{5}$ Sobre los encargos a Nicola Vaccaro (Nápoles, 1634-1709) para instituciones eclesiásticas napolitanas, ministros y barones particulares, tanto de la corte virreinal (como el regente marqués de Acerno, el consigliere Messía y el duque de Santa Severina) como en las provincias del Reame, así como su frustrada carrera como empresario del teatro de San Bartolomeo que a corto plazo arruinó su fama pictórica, vid. De Dominici, 1743, pp. 152-154.
} 
Nicola Vaccaro, di cui scrivendo la vita di Andrea suo padre abbiam[o] fatto parola, fu per qualche tempo scolaro ed amico di Salvatore in Napoli, e lo seguitò in Roma, dove studiò su l'opere sue, imitandone anche lo stile, e massimamente ne' tronchi; sicchè tornato poscià in Napoli, vendè alcuni de' suoi quadri per opere del Rosa. Ma poi ch'ebbe vedute l'opere perfettissime di Nicolò Pusino, ch'era amico di Salvatore, se n'invaghi a tal segno, che con la raccomandazione del Rosa ne divenne scolare, e fece tutte quelle pitture che nella vita di lui abbiamo mentovate in piè della vita di Andrea Vaccaro suo padre ${ }^{6}$.

Retomando la vida de su vástago, el dato más relevante aportado por el erudito Carreras se corresponde a la partida de matrimonio con Rosa Buffo, celebrado en Santa María del Mar el 16 de mayo de 1712. La señora, hija del comerciante italiano Francesco Buffo, había enviudado previamente de su primer marido, Giovanni Cosentino, de presumible origen napolitano ${ }^{7}$. Con respecto a la producción de Vaccaro, el autor catalán sólo advirtió su condición de pintor de Cámara del rey y la existencia de dos supuestos lienzos suyos representando la pareja real austriaca que, en 1902, pertenecían al conde de Belloch ${ }^{8}$.

Todas las referencias que a posteriori han aparecido sobre Andrea Vaccaro II sólo han repetido sin contrastar los datos de Carreras, por lo que la figura del pintor todavía permanece en el mayor de los silencios ${ }^{9}$. Sólo las pesquisas archivísticas pueden arrojar luz sobre don Andrea y su vinculación a la monarquía de Carlos III, indagándose no sólo su inserción en la corte barcelonesa, sino los medios de satisfacción de los gajes correspondientes a su persona como pintor de Cámara, oficio que años antes sirviera su compatriota Alessandro Castrioto ${ }^{10}$.

${ }^{6}$ De Dominici, 1743, p. 252.

${ }^{7}$ Carreras i Bulbena, 1902, p. 223. Los padrinos del enlace fueron el castellano Antonio de Acuña y el napolitano Tommaso Gargano. Gargano sirvió desde 1707 en la real guardia de Corps hasta serle concedida una patente de capitán de caballos en 1711. Su cercanía al monarca le hizo ser enviado a Nápoles para "ser empleado en goviernos regios" al año siguiente, para marchar temporalmente a Viena en 1715 con el objetivo de medrar en el cursus honorum napolitano. El capitán Gargano todavía vivía en Nápoles en 1725. Alcoberro (ed.), 2002, p. 122. Relación de todos los militares y demás sujetos que gozan diario. Sus grados, tiempo ha que siguen a S. M. C. C. y que llegaron a esta corte, Viena, 1725. Menos referencias documentales se conservan de Acuña, quien se exilió en 1713-1714 a los territorios cesáreos sin ningún miembro de su familia, y al que se le concedió una pensión, por ser de "la quarta clase de los españoles", de doscientos florines. Alcoberro (ed.), 2002, p. 29. Listado de exiliados según su cualidad, Viena, 1714; y p. 53. Listado de españoles que cobran pensión por Carlos VI, Viena, 1715

${ }^{8}$ Las dos obras se conservan en el Museu Palau Mercader, de Cornellà de Llobregat, con los números de inventario museístico 490 y 492 . No están firmados y, con gran probabilidad, el retrato ovalado de la joven Isabel Cristina provenga de un pincel centroeuropeo o flamenco, no del napolitano Vaccaro.

9 Alcolea, 1959-1960, p. 189; Vv. Aa., 1963; Urrea Fernández, 1977, pp. 233-234; Izzo, 2009, p. 17, nota 5. Incluso, una de las pocas obras conservadas de Andrea Vaccaro II, su autorretrato conservado en la iglesia partenopea de San Giovanni delle Monache, ha sido reiteradamente confundido con el pincel de su abuelo homónimo. La relación de autores que apoyan la atribución al primer Vaccaro se indica en Kiyomi Tuck-Scala, 2003, pp. 146, 162-164, 227 (reproducción de lienzo). La moda afrancesada que porta el retratado hace pensar en una datación muy posterior a la tradicionalmente expresada, pues su periodo de eclosión en Nápoles se sitúa entre 1690 y 1710. Sobre dicha cuestión cultural, vid. Frutos, 2009. Por último, también se ha adscrito a Vaccaro un lienzo conservado en el Kunsthistorisches Museum (inv. 7058) representando al joven monarca Carlos III con coraza ante un escenario clasicista y un paisaje bélico, que se ha considerado una alegoría de la toma de Barcelona en 1705. No obstante, la autoría del retrato ha sido tradicionalmente adscrita al flamenco Frans van Stampart, formado con Godfrey Kneller en Inglaterra y pintor de corte del monarca Habsburgo en Viena. La reciente vinculación a Vaccaro, en Morán Turina, 2010, pp. 179-184.

${ }^{10}$ La valía pictórica de Castrioto fue alabada por Giovanni Francesco Gemelli Careri en su conocido Giro del mondo. En una noticia de 8 de octubre de 1709, el viajero partenopeo informaba cómo los reyes habían partido a cazar en las afueras de Barcelona. Allí "[a la reina] la dovea ritrarre (come fece) un nostro napoletano, nomato Alessandro Castriotto: il quale come molto abile, e virtuoso nella miniatura, ebbe anche l'onore di far il ritratto del Re, dichiarandolo lor dipintore". Gemelli Careri, 1728, p. 65. Asimismo, sobre Castrioto y su vinculación con España, vid. Urrea Fernández, 1977, pp. 103-105. La existencia de una representación, de bella factura, de Carlos III durante su estancia española debido a la 
La llegada de Vaccaro de Nápoles a la España carolina debió producirse en el año 1710, cuando la junta de Italia consultó favorablemente su persona para ser admitido como "pintor de la Real Cámara" (24 de julio) ${ }^{11}$. El nombramiento consultado por el dicasterio italiano se producía sin atender al tradicional sistema de nóminas para este tipo de artistes de Cour de los Austrias madrileños o del propio Felipe $\mathrm{V}^{12}$. Similar alteración se viviría en agosto de 1711, con el nombramiento de Joseph Callejo y Aragón como escultor de Cámara mediante un más que probable desembolso pecuniario y sin mediar los jefes de palacio ${ }^{13}$. Por contra, al promocionarse a Joseph de Churriguera como trazador mayor de las obras reales en 1712, sí se otorgaron las órdenes administrativas pertinentes a su integración en la Casa Real al mayordomo mayor de la regente Isabel Cristina, conde de Cardona, y la junta de Obras y Bosques, el organismo palatino encargado de gestionar los pagos a tales oficiales regios desde comienzos del Seiscientos ${ }^{14}$.

La escasez de rentas catalanas para sostener la Casa del Rey movió a los ministros carolinos a promover a un segundo oficio al pintor Vaccaro para que obtuviera gajes suficientes con que sustentarse. Por despacho de 18 de agosto de 1710 se le concedió la alcaidía mayor de las cárceles de la Gran Corte della Vicaria de Nápoles. El cargo no debía servirse en persona, pues su producto serviría para proveer al artista las sumas necesarias para que "pudiera mantenerse en la corte". Es decir, habría de enajenarse a un tercero y recibir los réditos contantes que paliasen la falta de los gajes de Andrea Vaccaro en Barcelona ${ }^{15}$.

mano del napolitano Francesco Solimena -y que actualmente se conserva en el Museo di Capodimonte (Q1833)-puede aventurar un viaje fugaz del pintor partenopeo por la corte carolina. Por desgracia, frente al conocido contacto entre Solimena y Felipe V durante su estancia en Nápoles (1702), no se puede aseverar un encargo directo con el monarca Habsburgo hasta el conocido retrato grupal conservado en el Kunsthistorisches Museum (GG 1601), que corrió a cargo del conde Gundacker von Althann para la Galería Cesárea vienesa (1728). De Dominici, 1743, pp. 601-602.

${ }_{11}$ ASNa, Consiglio di Spagna, vol. 236, ff. 165r-166r. Decreto de Isabel Cristina de Braunschweig-Wolfenbüttel al mayordomo mayor y la junta del Bureo, Barcelona, 29 de julio de 1712.

${ }^{12}$ Para una mayor profundización sobre tal concepto y las posibilidades sociales y culturales de su estudio en la España borbónica, vid. Morales, 2007.

${ }_{13}$ AHN, Estado, legajo 8687. Título de Carlos III a favor de Joseph Callejo y Aragón, Barcelona, 25 de agosto de 1711. La posibilidad del nombramiento de Callejo por medio de un negociado venal se sugiere ante la notable cantidad de patentes y privilegios para los más diversos oficios regios durante los preparativos del viaje de Carlos III hacia Italia y el Sacro Imperio para recoger la herencia patrimonial de su hermano difunto, el emperador José I, y optar a la corona cesárea. Sobre tal coyuntura, vid. Quirós Rosado, 2015a, pp. 146-147.

${ }^{14}$ ASNa, Consiglio di Spagna, vol. 236, ff. 162r-163r. Decreto de Isabel Cristina de Braunschweig-Wolfenbüttel al mayordomo mayor y la junta de Obras y Bosques, Barcelona, 29 de julio de 1712. Los motivos de la concesión del cargo a Churriguera encarecen cómo el arquitecto había "seguido mi justa causa haviendo padezido por este motivo muchas persecuziones y abandonando vuestra cassa, familia y hazienda", al seguir al monarca hacia Barcelona, "a donde después el partido enemigo embió desterrada vuestra muger, y a los méritos que tenéis adquiridos en servicio del señor rey don Carlos Segundo (...) en el empleo de trazador mayor de las Obras Reales". ASNa, Consiglio di Spagna, vol. 236, ff. 162r163r. Decreto de Isabel Cristina de Braunschweig-Wolfenbüttel al mayordomo mayor y la junta de Obras y Bosques, Barcelona, 29 de julio de 1712. Sus bienes habían sido confiscados en 1710, ascendiendo a la modesta cantidad de 2.657 reales. León Sanz y Sánchez Belén, 1998, p. 154. El destierro de su esposa, que evocase el despacho regio, tuvo lugar en 1711, cuando Paula María Tafalla, fue expulsada de Castilla por orden de Felipe V, al igual que numerosas esposas de partidarios de Carlos III. AHPM, protocolo 14210 (escribano Francisco Pulido), ff. 209r-214v. Escritura de ajuste entre Alberto de Churriguera, Eugenio de Yuste y Antonio Álvarez, Madrid, 4 de diciembre de 1712. Tras la evacuación imperial de 1713 y el asedio de 1714, fechas aproximadas del final de su estancia en la urbe catalana, "y con el motivo de haverse restituido desde la ziudad de Barzelona a esta corte [madrileña]", Joseph de Churriguera contrató con Juan de Goyeneche la realización de las obras de Nuevo Baztán y el palacio madrileño del financiero navarro. Agulló y Cobo (ed.), 2005, p. 88. Asimismo, sobre Churriguera y las incógnitas de su etapa en Cataluña, vid. Blanco Esquivias, 2006, pp. 14-15.

${ }^{15}$ HHStA, Italien Spanischer Rat. Vorträge der Zentralbehörden, Karton 11. Consulta del consejo supremo de Italia, Barcelona, 30 de junio de 1712. No existe ninguna monografía sobre la venalidad napolitana durante el reinado de Carlos III. Para el periodo inmediatamente precedente, vid. Álvarez-Ossorio, 2007. 
Para su desgracia, Vaccaro no pudo percibir ningún beneficio del oficio. Éste había revertido a la Regia Camera napolitana por la filiación borbónica de su propietario (el madrileño Francisco Va1lejo), hasta que se adujo como propio por Andrea Avossa ${ }^{16}$. La falta de medios del pintor impidió la impugnación al solicitante, ni "emprender un pleyto"17. Recién desposado y falto de recursos para proseguir su estancia en Palacio, por memorial dirigido a la regente Isabel Cristina de BraunschweigWolfenbüttel en junio de 1712, don Andrea solicitó "una banca de mastrodatti", con facultad de sustitución, vaca en el Sacro Reale Consiglio por fallecimiento de Marcello Figliola y que interinamente ejercía un particular, Casimiro Scoppa. En caso que la soberana no condescendiese con la súplica, Vaccaro propuso otros cargos sin necesidad de ser servidos per se, trompeta de la Vicaria "y subastador del Sacro Real Consejo", que también habían sido devuelto al patrimonio regio por el fallecimiento de su antiguo poseedor, el marqués de Pontelatrone. La estimación económica que el pintor hacía de tales cargos ascendía a sesenta ducados mensuales "que es el subsidio que necesita para mantenerse en España sirviendo su plaza". Dicha cifra podría crecer si también se le remunerase con una ayuda pecuniaria "por una vez en tratas de vino u otros géneros en el Reyno de Nápoles".

La petición de Vaccaro fue evaluada por el consejo supremo de Italia que sustituyó, en el verano de 1711, a la anterior junta italiana de Barcelona. Para sus regentes, el empleo que se le concedió en la Vicaria se le podía asignar efectivamente, pues era "clara la razón que assiste a la parte contraria", mientras se cerraba la puerta a proveerle los otros solicitados en trueque. Más facilidad, tanto para el ministerio napolitano como para el propio consejo y el suplicante, radicaba en una mera ayuda pecuniaria: veinticinco ducados mensuales en Nápoles para que don Andrea subsistiese en Barcelona en caso que no se le conminase regresar a su ciudad natal, sumados a otros cuatrocientos ducados por una vez en tratas fiscales del Reame, suficientes para satisfacer lo devengado al artista desde su entrada al servicio carolino. Para desgracia del pintor de Cámara, la regente no se conformó con el parecer del consejo de Italia y solicitó información sobre "si esta parte [Vaccaro] desfruta otra gracia"18.

Posiblemente para solucionar los problemas subyacentes a la efectividad del oficio palatino, la soberana expidió en el mismo verano de 1712 un decreto a su mayordomo mayor y a la junta del Real Bureo. En él se ordenó asentar a Vaccaro en la planta de sirvientes de la Real Cámara. Tramitado el despacho por el consejo real de Castilla, se le concedieron a Vaccaro los gajes y privilegios de los demás homólogos suyos ${ }^{19}$. Se trataba de una completa novedad, ya que ninguno de los "pintores de Cámara" de la corte madrileña desde las primeras décadas del Seiscientos había visto situados sus salarios en dicho departamento palatino, sino en los haberes de la junta de Obras y Bosques ${ }^{20}$.

Mientras se solucionaba su financiación in situ, don Andrea volvió a insistir sobre la concesión de un oficio en Nápoles para subsistir en la corte austriaca. Un nuevo memorial tramitado por el

\footnotetext{
${ }^{16}$ Avossa gozaba de la futura de dicho cargo, con facultad de sustitución, por real privilegio de Carlos II, una vez vacase por el titular, Francisco Vallejo. AGS, Secretarías Provinciales, libro 288, f. 340r. Privilegio de Carlos II a favor de Andrea Avossa, Madrid, 14 de febrero de 1700. Sin embargo, posiblemente por cesión del propietario, Avossa aparece como carcelero mayor de la Vicaria ya en 1701. Durante la conjura del príncipe de Macchia, una turba saqueó su casa, junto con la del juez Antonio Plastena y los mismísimos tribunales supremos del Reame. Un anónimo recopilador de avisos lo refiere de esta manera: “(...) Così fecero alla casa di Andrea Avossa carceriere maggiore di detta Vicaria, che li levorno sino le porte delle finestre, che altro non se vedeva huomini, figlioli e femine carreiare robbe e processi di detti tribunali, chi materazzi, alcuni quadri, boffetti, e tutti chi una cosa e chi un'altra"; Luongo (ed.), 2003, p. 167.

${ }^{17}$ HHStA, Italien Spanischer Rat. Vorträge der Zentralbehörden, Karton 11. Consulta del consejo supremo de Italia, Barcelona, 30 de junio de 1712.

${ }^{18}$ Ibídem. El asiento de dicha consulta y resolución, en AHN, Estado, libro 1002, f. 100v.

19 ASNa, Consiglio di Spagna, vol. 236, ff. 165r-166r. Decreto de Isabel Cristina de Braunschweig-Wolfenbüttel al mayordomo mayor y la junta del Bureo, Barcelona, 29 de julio de 1712. En el decreto se especifica cómo Vaccaro ya había sufragado la caución de la media anata ante el escribano de cámara de Gobierno del citado consejo de Castilla.

${ }^{20}$ Sobre los pormenores de la financiación de los pintores de Cámara, vid. Martín González, 1958 y Vizcaíno Villanueva, 2005, pp. 319-329.
} 
consejo de Italia demuestra el notable conocimiento del pintor del oscuro mercado venal en Nápoles. Según sus noticias, la Regia Camera della Sommaria había procedido a la venta de la alcaidía que litigaba con Avossa "en 900 ducados (...) siendo assí que su valor es mucho más creçido conforme en otros memoriales tiene expuesto". Para contrarrestarlo, reclamó a la soberana la nulidad de la venta y su concesión efectiva, o su permuta por otro "offiçio equivalente", caso de "una banca" en el Sacro Regio Consiglio que había disfrutado hasta su muerte Niccolò d'Alessio "por acabarse en él la segunda vida" -vía sustituto-y la ayuda pecuniaria que solicitara con anterioridad. Viendo sus peticiones y la ausencia de concesiones previas, el consejo supremo de Italia consultó favorablemente la instancia de Vaccaro, ratificándose en la proposición hecha en junio, por la cual se satisfaría la merced en contante y "sin hazer mención de esta nueva súplica suya" ${ }^{21}$. La evaluación de los ministros regios se encaminó a la regente en los mismos términos ya expuestos, es decir, veinticinco ducados mensuales en Nápoles y cuatrocientos por una vez en tratas como ayuda de costa por su salario atrasado, todo ello aprobado por la soberana con un lacónico "Goze por ahora la merced en que le consulta el consexo"22.

Tras la publicación de la merced desaparece cualquier noticia sobre Andrea Vaccaro en la corte barcelonesa. Un informe elaborado a instancias del consejo supremo de España vienés en 1714 sitúa en Nápoles a Rosa Buffo, "viuda de don Andrés Vaccaro pintor de S. M. y que vino huyendo de Madrid", la cual había remitido un memorial al secretario Juan Antonio Alvarado para hacer presente sus necesidades pecuniarias al rey-emperador Carlos ${ }^{23}$. Por ello, bien en Barcelona antes de la salida de la regente y sus criados y ministros en la primavera de 1713, bien en la ciudad de Nápoles tras el retorno de los cortesanos partenopeos, la Parca recibió al pintor de la Real Cámara sin haber alcanzado los cincuenta años de edad.

\section{BIBLIOGRAFÍA}

Agulló y Cobo, Mercedes (ed.) (2005): Documentos para la historia de la escultura española, Madrid, Fundación de Apoyo a la Historia del Arte Hispánico.

Albareda Salvadó, Joaquim (2010): La guerra de Sucesión de España (1700-1714), Barcelona, Crítica.

Alcoberro, Agustí (ed.) (2002): L'exili austriacista (1713-1747), tomo II, Barcelona, Fundació Noguera.

Alcolea, Santiago (1959-1960): La pintura en Barcelona durante el siglo XVIII. En: Anales y boletín de los museos de arte de Barcelona, 14-15.

Álvarez-Ossorio Alvariño, Antonio (2007): La venta de magistraturas en el Reino de Nápoles durante los reinados de Carlos II y Felipe V. En: Chronica nova, 33, pp. 57-94.

Badosa i Coll, Elisa (2003): Els capitals d'Itàlia a la cort de l'Arxiduc Carles d'Àustria a Barcelona. En: Pedralbes, 23/2, pp. 233-252.

Bernardini, Laura (2009a): Ferdinando Galli Bibiena alla corte di Barcellona e la scenografia per la Festa della Peschiera. En: Quaderns d'Italià, 14, pp. 131-158.

Bernardini, Laura (2009b): Teatro e musica a Barcellona alla corte di Carlo III d'Asburgo. En: Recerca Musicologica, 19, pp. 199-227.

Blanco Esquivias, Beatriz (2006): «Ni fatuos ni delirantes”. José Benito Churriguera y el esplendor del barroco español. En: Lexicon, 2, pp. 6-23.

${ }^{21}$ HHStA, Italien Spanischer Rat. Vorträge der Zentralbehörden, Karton 12. Acuerdo del consejo supremo de Italia, s. 1., s. f.; Barcelona, octubre de 1712.

${ }^{22}$ Los problemas de la concesión efectiva del cargo a Vaccaro pudieron provenir de su venta, por novecientos ducados, realizada por la Regia Camera della Sommaria al citado Avossa. HHStA, Italien Spanischer Rat. Vorträge der Zentralbehörden, Karton 12. Consulta del consejo supremo de Italia, Barcelona, 6 de octubre de 1712. El decreto regio se publicó en 20 de octubre de 1712. Asimismo, el regesto de la consulta y la orden de la soberana se encuentran en AHN, Estado, libro 1002, f. 117v.

${ }^{23}$ HHStA, Italien Spanischer Rat. Vorträge der Zentralbehörden, Karton 18. Informe del consejo supremo de España, s. 1., s. f.; Viena, 1714. 
Boix, Vicente (1845): Historia de la ciudad y reino de Valencia, tomo I, Valencia, Imprenta de don Benito Monfort.

Carreras i Bulbena, Joseph Rafael (1902): Carlos d'Austria y Elisabeth de Brunswich Wolfenbüttel a Barcelona y Girona. Musiques, festes, càrrechs palatins, defensa de l'emperador, religiositat d'aquest monarques, Barcelona, Tip. L'Avenç.

De Dominici, Bernardo (1743): Vite de' pittori, scultori, ed architetti napoletani, tomo III, Nápoles, per Francesco e Cristoforo Ricciardi, stampatori del Real Palazzo.

Frutos, Leticia de (2009): «Virtuosos of the Neapolitan opera in Madrid: Alessandro Scarlatti, Matteo Sassano, Petruccio and Filippo Schor«. En: Early Music, 37/2, pp. 187-200.

García Espuche, Albert (2014): Una societat assetjada. Barcelona, 1713-1714, Barcelona, Empùries.

Gemelli Careri, Giovanni Francesco (1728): Giro del mondo del dottor D. Gio Francesco Gemelli Careri. Nuova edizione accresciuta, ricorretta, e divisa in nove volumi, tomo IX, Venecia, presso Sebastiano Coleti.

Izzo, Mariaclaudia (2009): Nicola Vaccaro (1640-1709): un artista a Napoli tra Barocco e Arcadia, Todi, Tau editrice.

Kalmár, János (1998): Sobre la cort barcelonina de l'Arxiduc Carles d'Áustria. En: Pedralbes, 18/2, pp. 299-302.

Kiyomi Tuck-Scala, Anna (2003): The documented paintings and life of Andrea Vaccaro (1604-1670). Tesis doctoral inédita, State College, Penn.: The Penssylvania State University.

León Sanz, Virginia (2014): El Archiduque Carlos y los austracistas, Sant Cugat del Vallés, Arpegio.

León Sanz, Virginia / Sánchez Belén, Juan Antonio (1998): Confiscación de bienes y represión borbónica en la Corona de Castilla a comienzos del siglo XVIII. En: Cuadernos de Historia Moderna, 21, pp. 127-175.

Lipp, Danièle (2007): "Músicos italianos entre las cortes de Carlos III/VI en Barcelona y Viena". En La pérdida de Europa. La guerra de Sucesión por la Monarquía de España; Álvarez-Ossorio Alvariño, Antonio / García García, Bernardo J. / León Sanz, Virginia (eds.), Madrid: Fundación Carlos de Amberes, pp. 159-179.

Luongo, Dario (ed.) (2003): Diario napolitano dal 1700 al 1709, Nápoles, Società Napoletana di Storia Patria.

Martín González, Juan José (1958): Arte y artistas del siglo XVII en la Corte. En: Archivo Español de Arte, XXXI/122, pp. 125-142.

Morales, Nicolas (2007): L'artiste de cour dans l'Espagne du XVIIle siècle: étude de la communauté des musiciens au service de Philippe V (1700-1746), Madrid-Burdeos, Casa de Velázquez.

Morán Turina, José Miguel (2010): “Felipe V de Habsburgo?”. En: Sevilla y corte: las artes y el lustro real (1729-1733); Morales, Nicolas / Quiles García, Fernando (eds.), Madrid-Burdeos: Casa de Velázquez, pp. 179-184.

Pascual Chenel, Álvaro (2010): El retrato de Estado durante el reinado de Carlos II. Imagen y propaganda, Madrid, Fundación Universitaria Española.

Quirós Rosado, Roberto (2015a): Constantia et fortitudine. La corte de Carlos III y el gobierno de Italia, 1706-1714. Tesis doctoral inédita, Madrid, Universidad Autónoma de Madrid.

Quirós Rosado, Roberto (2015b): "Reconstruyendo el ceremonial. Diplomacia y audiencias públicas en la corte carolina de Barcelona". En: Actes del Congrés El Tractats d'Utrecht. Clarors i foscors de la pau. La resistència dels catalans; Mollfulleda, Conxita y Sallés, Núria (eds.), Barcelona: Museu d'Història de Catalunya. Generalitat de Catalunya. Departament de Cultura, pp. 473-478.

Sommer-Mathis, Andrea (2007): "Música y teatro en las cortes de Madrid, Barcelona y Viena durante el conflicto dinástico Habsburgo-Borbón. Pretensiones políticas y teatro cortesano". En: La pérdida de Europa. La guerra de Sucesión por la Monarquía de España; Álvarez-Ossorio Alvariño, Antonio / García García, Bernardo J. / León Sanz, Virginia (eds.), Madrid: Fundación Carlos de Amberes, pp. 181-198.

Úbeda de los Cobos, Andrés (2002): "Felipe V y el retrato de corte". En: El arte en la corte de Felipe V; Morán Turina, Miguel (ed.), Madrid: Fundación Caja de Madrid. Patrimonio Nacional. Museo Nacional del Prado, pp. 89-140.

Urrea Fernández, Jesús (1977): La pintura italiana del siglo XVIII en España, Valladolid: Universidad de Valladolid.

Vizcaíno Villanueva, María A. (2005): El pintor en la sociedad madrileña durante el reinado de Felipe IV, Madrid, Fundación Universitaria Española.

Voltes Bou, Pere (1963): Barcelona durante el gobierno del Archiduque Carlos de Austria (1705-1714), tomo I, Barcelona, Instituto Municipal de Historia.

VV. AA. (1993): Barock in Neapel: Kunst zur Zeit der österreichischen Vizekönige, Nápoles, Electa Napoli.

Abreviaturas:

AGS. Archivo General de Simancas (Simancas).

AHN. Archivo Histórico Nacional (Madrid).

AHPM. Archivo Histórico de Protocolos de Madrid (Madrid).

ASNa. Archivio di Stato di Napoli (Nápoles).

HHStA. Haus-, Hof- und Staatsarchiv (Viena).

Fecha de recepción: 27-IV-2015

Fecha de aceptación: 13-I-2016 